\title{
An Enumeration Problem for a Congruence Equation*
}

\author{
Richard A. Brualdi** and Morris Newman \\ Institute for Basic Standards, National Bureau of Standards, \\ Washington, D.C. 20234
}

(September 9, 1969)

It is shown that the number of $n$-tuples $\left(x_{0}, x_{1}, \ldots, x_{n-1}\right)$ of nonnegative integers such that

$$
\begin{gathered}
\sum_{i=0}^{n-1} x_{i}=n, \\
\sum_{i=0}^{n-1} i x_{i} \equiv 0 \bmod n,
\end{gathered}
$$

is given by

$$
\frac{1}{n} \sum_{d \mid n}\left(\begin{array}{c}
2 d-1 \\
d
\end{array}\right) \varphi\left(\frac{n}{d}\right)
$$

Key words: Circulants; congruences; permanents.

\section{Introduction}

In 1952 M. Hall, Jr. proved the following theorem ${ }^{1}$ (see footnote 1): If $G$ is a finite abelian group of order $n$ with elements $a_{1}, a_{2}, \ldots, a_{n}$, and $c_{1}, c_{2}, \ldots, c_{n}$ are $n$ (not necessarily distinct) elements of $G$, then there exists a permutation $\sigma$ of $\{1,2, \ldots, n\}$ such that the differences $a_{\sigma(1)}-a_{1}, a_{\sigma(2)}-a_{2}, \ldots, a_{\sigma(n)}-a_{n}$ are $c_{1}, c_{2}, \ldots, c_{n}$ in some order, if and only if

$$
\sum_{i=1}^{n} c_{i}=0
$$

The necessity of (1) is trivial, and Hall gives an elegant proof that condition (1) implies the existence of such a permutation $\sigma$. If $G$ is the cyclic group of order $n$, then Hall's theorem may be rephrased in terms of congruences as follows: Let $x_{0}, x_{1}, \ldots, x_{n-1}$ be $n$ nonnegative integers with

$$
\sum_{i=0}^{n-1} x_{i}=n
$$

Then there is a permutation $\sigma$ of $\{1,2, \ldots, n\}$ such that

$$
\sigma(i)-i \equiv k \quad(\bmod n)
$$

has exactly $x_{k}$ solutions in $i, 1 \leqslant i \leqslant n$, for each $k=0,1 \ldots, n-1$ if and only if

$$
0 x_{0}+1 x_{1}+\ldots+(n-1) x_{n-1} \equiv 0(\bmod n) .
$$

*Partially supported by N.S.F. Grant No. GP-7073.

**Present address: Department of Mathematics, University of Wisconsin, Madison, Wis. 53706.

'M. Hall, Jr., A Combinatorial Problem on Abelian Groups, Proc. A. M. S. $584-587$ (1952). 
The purpose of this note is to count the number of solutions of (2) in nonnegative integers $x_{i}$ with $\sum_{i=0}^{n-1} x_{i}=n$. An application to the permanent of a circulant is given.

\section{Main Result}

The motivation having been given, we may now state and prove our main result.

Theorem: Let $\mathrm{n}$ be a positive integer. Let $\mathrm{F}(\mathrm{n})$ be the number of $n$-tuples $\left(\mathrm{x}_{0}, \mathrm{x}_{1}, \ldots ., \mathrm{x}_{\mathrm{n}-1}\right)$ satisfying:

$$
\begin{aligned}
x_{i} \geqslant 0,(i=0,1, \ldots, n-1) & \\
& \sum_{i=0}^{n-1} x_{i}=n, \\
& \sum_{i=0}^{n-1} i x_{i} \equiv 0(\bmod n) .
\end{aligned}
$$

Then

$$
\mathrm{F}(\mathrm{n})=\frac{1}{\mathrm{n}} \sum_{\mathrm{d} \mid \mathrm{n}}\left(\begin{array}{c}
2 \mathrm{~d}-1 \\
\mathrm{~d}
\end{array}\right) \varphi\left(\frac{\mathrm{n}}{\mathrm{d}}\right)
$$

where the summation extends over all positive integers $\mathrm{d}$ dividing $\mathrm{n}$, and where $\varphi$ is Euler's function.

Proof: The proof uses generating functions. Define

$$
f_{n}(w, z)=\left[(1-z)(1-w z) \ldots\left(1-w^{n-1} z\right)\right]^{-1} .
$$

Then

$$
f_{n}(w, z)=\left(\sum_{k=0}^{\infty} z^{k}\right)\left(\sum_{k=0}^{\infty} w^{k} z^{k}\right) \ldots\left(\sum_{k=0}^{\infty} w^{k(n-1)} z^{k}\right)
$$

and it is clear that $F(n)$ is the sum of the coefficients of $z^{n} w^{n t}, 0 \leqslant t \leqslant n-1, \operatorname{in} f_{n}(w, z)$. Write

$$
f_{n}(w, z)=\sum_{k=0}^{\infty} B_{k} z^{k} \quad\left(B_{k}=B_{k}(n, w)\right)
$$

Then because

$$
f_{n+1}(w, z)=\frac{f_{n}(w, z)}{1-w^{n} z}
$$

and

we obtain

$$
f_{n}(w, w z)=\left[(1-w z)\left(1-w^{2} z\right) \ldots\left(1-w^{n} z\right)\right]^{-1}
$$

Thus

$$
f_{n}(w, w z)=(1-z) f_{n+1}(w, z)=\frac{1-z}{1-w^{n} z} f_{n}(w, z)
$$

so that

$$
\sum_{k=0}^{\infty} B_{k} w^{k} z^{k}=\frac{1-z}{1-w^{n} z} \sum_{k=0}^{\infty} B_{k} z^{k}
$$

$$
\sum_{k=0}^{\infty} B_{k} w^{k} z^{k}-\sum_{k=0}^{\infty} B_{k} w^{n+k} z^{k+1}=\sum_{k=0}^{\infty} B_{k} z^{k}-\sum_{k=0}^{\infty} B_{k} z^{k+1} .
$$

Hence for $k \geqslant 1$,

or

$$
B_{k} w^{k}-B_{k-1} w^{n+k-1}=B_{k}-B_{k-1},
$$

$$
B_{k}=\frac{1-w^{n+k-1}}{1-w^{k}} B_{k-1} \quad(k \geqslant 1) .
$$


Thus since $B_{0}=1$,

$$
B_{k}=\prod_{r=1}^{k} \frac{1-w^{n+r-1}}{1-w^{r}} \quad(k \geqslant 0),
$$

an empty product being 1 . Therefore

$$
f_{n}(w, z)=\sum_{k=0}^{\infty}\left\{\prod_{r=1}^{k} \frac{1-w^{n+r-1}}{1-w^{r}}\right\} z^{k}
$$

and $F(n)$ is the sum of the coefficients of $w^{n t}, 0 \leqslant t \leqslant n-1$, in

$$
g_{n}(w)=\prod_{r=1}^{n} \frac{1-w^{n+r-1}}{1-w^{r}}=\prod_{r=1}^{n-1} \frac{1-w^{n+r}}{1-w^{r}}
$$

Now, $g_{n}(w)$ is a polynomial in $w$ of degree $\sum_{r=1}^{n-1}\{n+r-r\}=n(n-1)$, and has nonnegative coefficients (since $f_{n}(w, z)$ has nonnegative coefficients). Since

$$
\sum_{\zeta: \zeta^{n}=1} \zeta^{k}=\left\{\begin{array}{l}
n, \text { if } n \text { divides } k \\
0, \text { otherwise }
\end{array}\right.
$$

we have

$$
n F(n)=\sum_{\zeta: \zeta^{n}=1} g_{n}(\zeta)
$$

the summations extending over all $n$th roots of unity.

Suppose now that $\zeta$ is a primitive $d$ th root of unity, where $d \mid n$. Since

$$
\lim _{w \rightarrow \zeta} \frac{1-w^{n+r}}{1-w^{r}}=\left\{\begin{array}{l}
\frac{n+r}{r}, \text { if } d \text { divides } r \\
1, \text { otherwise }
\end{array}\right.
$$

we have that

$$
g_{n}(\zeta)=\prod_{\substack{1 \leq r \leq n-1 \\
r=0 \bmod d}} \frac{n+r}{r}=\prod_{s=1}^{\frac{n}{d}-1} \frac{n+s d}{s d}=\left(\begin{array}{c}
2 \frac{n}{d}-1 \\
\frac{n}{d}
\end{array}\right) .
$$

Therefore, since there are $\varphi(d) n$th roots of unity which are primitive $d$ th roots of unity,

$$
\begin{aligned}
F(n) & =\frac{1}{n} \sum_{d \mid n}\left(\begin{array}{c}
2 \frac{n}{d}-1 \\
\frac{n}{d}
\end{array}\right) \varphi(d) \\
& =\frac{1}{n} \sum_{d \mid n}\left(\begin{array}{c}
2 d-1 \\
d
\end{array}\right) \varphi\left(\frac{n}{d}\right) .
\end{aligned}
$$

This proves the theorem.

\section{An Application}

Let $A=\left[a_{i j}\right]$ be an $n \times n$ matrix. If $\sigma$ is a permutation of $\{1,2, \ldots, n\}$ then

$$
a_{1 \sigma(1)} a_{2 \sigma(2)} \ldots a_{n \sigma(n)}
$$


is called a diagonal product of $A$. The permanent of $A$, denoted by per $(A)$, is the sum of the diagonal products of $A$. Thus

$$
\operatorname{per}(A)=\sum_{\sigma} a_{1 \sigma(1)} a_{2 \sigma(2)} \ldots a_{n \sigma(n)}
$$

the summation extending over all permutations of $\{1,2, \ldots, n\}$. Suppose $A$ is the $n$ by $n$ circulant

$$
\left[\begin{array}{cccc}
a_{0} & a_{1} & \ldots & a_{n-1} \\
a_{n-1} & a_{0} & \ldots & a_{n-2} \\
\cdot & \cdot & & \cdot \\
a_{1} & a_{2} & \ldots & a_{0}
\end{array}\right] .
$$

Then the diagonal product $a_{1 \sigma(1)} a_{2 \sigma(2)} \ldots a_{n \sigma(n)}$ equals $a_{0}^{x} a_{1}^{x} \ldots \ldots a_{n-1}^{x_{n-1}}$ where $x_{k}$ is the number of integers $i, 1 \leqslant i \leqslant n$, such that $\sigma(i)-i \equiv k(\bmod n)$.

By Hall's theorem, if $x_{0}, x_{1}, \ldots ., x_{n-1}$ are integers satisfying the hypothesis of the theorem, then $a_{0}^{x_{0}} a_{1}^{x_{1}} \ldots a_{n-1}^{x_{n-1}}$ is a diagonal product of the circulant $A$. Thus we have the following corollary.

CoROLlaRY: The number of formally distinct diagonal products of an $\mathrm{n}$ by $\mathrm{n}$ circulant is given by

$$
\frac{1}{\mathrm{n}} \sum_{\mathrm{d} \mid \mathrm{n}}\left(\begin{array}{c}
2 \mathrm{~d}-\mathrm{l} \\
\mathrm{d}
\end{array}\right) \varphi\left(\frac{\mathrm{n}}{\mathrm{d}}\right)
$$

Some other results on the permanent of a circulant are given by the authors in the reference below. ${ }^{2}$

(Paper 74B1-315)

\footnotetext{
${ }^{2}$ R. A. Brualdi and M. Newman, Some Theorems on the Permanent, J. Res. Nat. Bur. Stand. (U.S.), 69B (Math. Sci.) No. 3, 159-163 (July-Oct. 1965).
} 